\title{
Electrostatic Waves in a Maxwellian Dusty Plasma with Variable Charge on Dust Particles
}

\author{
R. S. Schneider, L. F. Ziebell, \\ Instituto de Física, Universidade Federal do Rio Grande do Sul, \\ Caixa Postal 15051, CEP 91501-970, Porto Alegre, RS, Brazil. \\ M. C. de Juli, and V. Jatenco-Pereira \\ Instituto de Astronomia, Geofísica e Ciências Atmosféricas, Universidade de São Paulo, \\ Rua do Matão 1226, Cidade Universitária, CEP 05508-900, São Paulo, SP, Brazil
}

Received on 13 April, 2006

\begin{abstract}
A kinetic formulation developed to analyze wave propagation in dusty plasmas, which takes into account the variation of the charge of the dust particles due to inelastic collisions with electrons and ions, is utilized to study the propagation and damping of electrostatic waves with wave number exactly parallel to the external magnetic field and Maxwellian distributions for the electrons and ions in the equilibrium. It is shown that, due to the presence of the dust, the damping of Langmuir waves in the region of large wavelengths is increased as compared to conventional Landau damping. Langmuir waves in the occurrence of collisional charging of dust particles also feature weak damping for small wavelengths, which vanishes if the effect of collisional charging of the dust particles is neglected in the dispersion relation. It is also shown that the damping of ion-acoustic waves is modified by the presence of the dust, and that some damping effect due to the dust particles remains even if the effect of collisional charging of dust particles is neglected in the dispersion relation.
\end{abstract}

Keywords: Langmuir waves; Electrostatic waves; Kinetic theory; Magnetized dusty plasmas; Dust charge fluctuation; Wave propagation.

\section{INTRODUCTION}

Waves and instabilities in dusty plasmas have attracted a great deal of interest in the last few years, since the presence of the dust particles may significantly modify the normal modes of oscillation of the plasma, as well as originate new modes of ultra-low frequency associated to the dynamics of the dust particles.

As a consequence of this interest, there is a respectable amount of theoretical work on waves and instabilities in dusty plasmas, particularly on electrostatic waves, which may be traced back to the pioneer work by Bliokh and Yarashenko [1] about waves in Saturn's rings. Most of these published works utilized fluid theory to describe the dusty plasmas, and only a few of them take into account the collisional charging of the dust particles [2, 3], although the importance of this effect to the propagation and damping of the waves is already well known $[4,5]$.

Some of the studies on electrostatic waves have predicted the instability of longitudinal oscillations due to the dust charge variations [6, 7], introducing ad hoc hypothesis related to the charging dynamics of the dust particles. However, a self-consistent formulation for the charge dynamics, using fluid theory, has been proposed by Bhatt and Pandey [8], and applied by Bhatt to the case of Langmuir waves [2]. These works have shown that the longitudinal oscillations are indeed subject to damping instead of growth, when the charging dynamics of the dust charge is appropriately taken into account, in a self-consistent manner. Self-consistent approaches to the dynamic of the dust charge variations can also be found in the works by D'Angelo [3] and by Vladimirov et al. [9].

Although very important studies have been conducted us- ing an hydrodynamical approach, it must be recognized that the fluid formulation has an important limitation: it can not describe purely kinetic effects such as the Landau damping. This consideration, per se, offers motivation for kinetic studies on dusty plasmas. Moreover, it has been shown that the dust charging process must be included in a kinetic approach, for proper derivation of the wave damping [10]. The reason is basically that the charging process is one of the most conspicuous and important dissipative processes to occur in a dusty plasma. As argued in Ref. [11], it is not possible to separate the conventional Landau damping and the damping due to the interaction of ions and electrons with the dust particles, at least for ion-acoustic waves.

The kinetic approach has been used in some investigations, as in the study of instabilities of ion-acoustic waves produced by current in a collisionless dusty plasma, completely ionized, appearing in the paper by Rosenberg [12]. This work has established a critical drift velocity of electrons, relative to the ions, for the occurrence of the instability. However, effects of the variation of the electric charge of the dust particles have not been taken into account.

Experimental studies on electrostatic waves have also been systematically conducted along the last few years. Some of these were directed to the investigation of low-frequency waves $\left(\omega<\Omega_{i}\right)$, such as the so-called ion-acoustic waves [13], while others aimed to the investigation of ultra lowfrequency waves $\left(\omega<\left|\Omega_{d}\right|\right)$, characterizing the mode known as the dust-acoustic wave [14].

As an example of experiment on ion-acoustic waves, it can be mentioned that conducted by Barkan et al. [15], which has shown that the phase velocity of the ion-acoustic waves increases with the density of negatively charged dust grains, ul- 
timately leading to the decrease of Landau damping for these waves. Another experiment by the same group of researchers has investigated the excitation of ion-acoustic waves by currents in a dusty plasma [16].

A kinetic description has been adopted by ourselves in a recent publication, utilized to analyze the propagation of electromagnetic waves in dusty plasmas, taking into account the variation of the dust charges due to inelastic collisions with electrons and ions [17].

In the formulation utilized in Ref. [17], the components of the dielectric tensor depend on the frequency of inelastic collisions of plasma electrons and ions with the dust particles. This collision frequency is momentum dependent, and, in order to more easily evaluate momentum integrals and solve the dispersion relation, we have utilized the simplifying assumption that the momentum dependent value of the inelastic collision frequency is replaced by a frequency averaged over the electron or ion distribution function, respectively. The formulation has been up to now applied to the particular case of low-frequency waves propagating along the ambient magnetic field, incorporating many details which have appeared in previous publications [17-19].

In the present paper, we resume the use of the same basic kinetic formulation, applied to the study of electrostatic waves propagating along the ambient magnetic field. Using this approach, we obtain the dispersion relation and the damping rates for electrostatic waves. By suitable choice of the frequency interval, we study the cases of Langmuir waves and of ion-acoustic waves, in the presence of dust, keeping effects due to the charging of the dust particles due to inelastic collisions with electrons and ions. The damping rates obtained for these waves consequently include the conventional Landau damping and the damping associated to the incorporation of electrons and ions by the dust particles.

The structure of the paper is the following. In Section II we briefly outline the model used to describe the dusty plasma. In Section III we present essential features of the dielectric tensor to be used in the discussion of wave propagation exactly parallel to the external magnetic field, derived assuming Maxwellian distributions for the electrons and ions in the equilibrium, and the ensuing dispersion relation for electrostatic waves. In Sections IV and V the numerical results obtained from the dispersion relation modified by the dust for the cases of Langmuir and ion-acoustic waves are presented and discussed. The conclusions are presented in Section VI. Appendixes $\mathrm{A}$ and $\mathrm{B}$ are included, respectively providing additional details of the formulation used in the present paper and comparing this formulation with other well-known approach found in the literature.

\section{THE DUSTY PLASMA MODEL}

In our general kinetic formulation we consider a plasma in a homogeneous external magnetic field $\mathbf{B}_{\mathbf{0}}=B_{0} \mathbf{e}_{\mathbf{z}}$. In this magnetized plasma we take into account the presence of spherical dust grains with constant radius $a$ and variable charge $q_{d}$; this charge originates from inelastic collisions between the dust particles and particles of species $\beta$ (electrons and ions), with charge $q_{\beta}$ and mass $m_{\beta}$. For simplicity, we will consider simply charged ions.

The charging model for the dust particles must in principle take into account the presence of an external magnetic field. This field should influence the characteristics of the charging of dust particles, because the path described by electrons and ions is modified: in this case we have cyclotron motion of electrons and ions around the magnetic field lines. However, it has been shown by Chang and Spariosu [20], through numerical calculation, that for $a \ll \rho_{G}$, where $\rho_{G}=(\pi / 2)^{1 / 2} r_{L e}$ and $r_{L e}$ is the electron Larmor radius, the effect of the magnetic field on the charging of the dust particles can be neglected. For the values of parameters used in the present work the relation $a \ll \rho_{G}$ is always satisfied.

We will consider that the dust grain charging process occurs by the capture of plasma electrons and ions during inelastic collisions between these particles and the dust particles. Since the electron thermal speed is much larger than the ion thermal speed, the dust charge will be preferentially negative. As a cross-section for the charging process of the dust particles, we use expressions derived from the OML theory (orbital motion limited theory) [21, 22].

In the present work we focus our attention on weakly coupled dusty magneto-plasmas, in which the electrostatic energy of the dust particles is much smaller than their kinetic energy. This condition is not very restrictive, since a large variety of natural and laboratory dusty plasmas can be classified as weakly coupled [23].

Dust particles are assumed to be immobile, and consequently the validity of the proposed model will be restricted to waves with frequency much higher than the characteristic dust frequencies. In particular we will consider the regime in which $\left|\Omega_{d}\right| \ll \omega_{p d}<\omega$, where $\omega_{p d}$ and $\Omega_{d}$ are the plasma frequency and the cyclotron frequency of the dust particles, respectively. This modeling excludes the modes that can arise from the dust dynamics.

As we will see, in this range of frequencies the dust particles modify the dispersion relation, through modifications of the quasi-neutrality condition and through effects due to the charging of the dust particles by inelastic collisions with ions and electrons. These effects due to the dust charging can provide an additional mechanism for the wave damping, beyond the well-known Landau damping mechanism.

\section{DISPERSION RELATION FOR ELECTROSTATIC WAVES IN THE CASE OF MAXWELLIAN DISTRIBUTION FUNCTIONS}

The dielectric tensor for a magnetized dusty plasma, homogeneous, fully ionized, with identical immobile dust particles and charge variable in time, can be written in the following way

$$
\varepsilon_{i j}=\varepsilon_{i j}^{C}+\varepsilon_{i j}^{N}
$$

where the explicit expressions for $\varepsilon_{i j}^{C}$ and $\varepsilon_{i j}^{N}$ are given in Refs. [17-19] and also in Appendix A of the present work. 
The components $\varepsilon_{i j}^{C}$ have a term which is formally identical to the components of the dielectric tensor of a magnetized homogeneous conventional plasma of electrons and ions, but with the resonant denominator modified by the addition of a purely imaginary factor which contains the inelastic collision frequency of electrons and ions with the dust particles. There is another term, appearing only in the components $\varepsilon_{i 3}^{C}$, which is added to the term obtained with the prescription above, which is proportional to the same inelastic collision frequency of electrons and ions with the equilibrium population of dust particles. If $f_{\beta 0}$ is a Maxwellian distribution the effects due to the dust charging by collisions with electrons and ions appear in the $\varepsilon_{i j}^{C}$ components only in the resonant denominator, being this result independent of the direction of $\mathbf{k}$.

The term $\varepsilon_{i j}^{N}$ is entirely new and only occurs in the presence of dust particles with variable charge. Its form is strongly dependent of the model used to describe the charging process of the dust particles. It is a non additive part of the response function, since it is composed as a product of integral factors which depend on the equilibrium distribution functions, as shown by Eq. (A8) of Appendix A. This point is also discussed in Appendix B, where the electrostatic case is considered with more details, including comparison with other formulation found in the literature. In the case of propagation exactly parallel to the external magnetic field, the term $\varepsilon_{i j}^{N}$ appearing in Eq. (1) is non-vanishing only for $i=j=3$, regardless of the detailed form of the distribution function $f_{\beta 0}$.

In the case of propagation parallel to the external magnetic field and Maxwellian distributions for electrons and ions, the 13 and 23 components of the dielectric tensor vanish, and the dispersion relation therefore follows from the determinant

$$
\operatorname{det}\left(\begin{array}{ccc}
\varepsilon_{11}^{C}-N_{\|}^{2} & \varepsilon_{12}^{C} & 0 \\
-\varepsilon_{12}^{C} & \varepsilon_{11}^{C}-N_{\|}^{2} & 0 \\
0 & 0 & \varepsilon_{33}^{C}+\varepsilon_{33}^{N}
\end{array}\right)=0
$$

In this expression, $N_{\|}=k_{\|} c / \omega$ is the refractive index in the direction parallel to the external magnetic field. The dispersion relation for electrostatic waves is obtained by considering the case of $E_{z} \neq 0$, which means

$$
\varepsilon_{33}=\varepsilon_{33}^{C}+\varepsilon_{33}^{N}=0
$$

As an initial approach to this dispersion relation, we neglect the effect of the term $\varepsilon_{33}^{N}$, which is entirely due to the fluctuation of the charge of the dust particles. Some effects due to the charging of the dust particles, however, remain in the term $\varepsilon_{33}^{C}$, and therefore an approximated dispersion relation can be written as follows,

$$
\varepsilon_{33}^{C}=1+\sum_{\beta} X_{\beta} \hat{I}_{\beta}^{0}=0
$$

where

$$
\begin{aligned}
X_{\beta} & =\frac{\omega_{p \beta}^{2}}{\omega^{2}}, \omega_{p \beta}^{2}=\frac{4 \pi n_{\beta 0} q_{\beta}^{2}}{m_{\beta}}, \\
\widehat{I}_{\beta}^{0} & =\frac{\omega}{n_{\beta 0}} \int d^{3} p \frac{p_{\|} \partial f_{\beta 0} / \partial p_{\|}}{\omega-k_{\|} p_{\|} / m_{\beta}+i v_{\beta d}^{0}(p)}, \\
v_{\beta d}^{0}(p) & =\frac{\pi a^{2} n_{d 0}}{m_{\beta}} \frac{\left(p^{2}+C_{\beta}\right)}{p} H\left(p^{2}+C_{\beta}\right), \\
C_{\beta} & =-\frac{2 q_{\beta} m_{\beta} q_{d 0}}{a} .
\end{aligned}
$$

The subscript $\beta=e, i$ identifies electrons and ions respectively, $q_{d 0}=\varepsilon_{d} e Z_{d}$ is the equilibrium charge of the dust particles (positive, $\varepsilon_{d}=+1$, or negative, $\varepsilon_{d}=-1$ ) and $H$ denotes the Heaviside function. The number of charges in each dust particle, $Z_{d}$, is calculated from the equation of balance of currents in the dust particles, in the equilibrium state, and from the quasi-neutrality condition, which gives also $n_{e 0}$ if we fix the ion and dust densities $n_{i 0}$ and $n_{d 0}$. It is noticed that this dispersion relation does not feature any effect due to the magnetic field, being exactly the same as the dispersion relation for electrostatic waves in unmagnetized dusty plasma, as in the case of dustless plasmas.

The effect of the dust particles on the dispersion relation, given by Eq. (4), occurs via the quasi-neutrality condition $\left(n_{i 0} \neq n_{e 0}\right)$, and also via the terms which contain the inelastic collision frequency $v_{\beta d}^{0}(p)$. Following the same procedure used in Ref. [17], in order to simplify the evaluation of the integral $\hat{I}_{\beta}^{0}$ we replace the functions $v_{\beta d}^{0}(p)$ by their average values in momentum space,

$$
v_{\beta} \equiv \frac{1}{n_{\beta 0}} \int d^{3} p v_{\beta d}^{0}(p) f_{\beta 0} .
$$

This approximation is adopted in order to arrive at a relatively simple estimate of the effect of the charging of dust particles due to collisions with electrons and ions, effect which is frequently neglected in analysis of the dispersion relation for waves in dusty plasmas. If significant effects are obtained due to this collisional charging there will be reason for further research in order to improve the approximation. The approximation will provide at least qualitatively a fair idea about the effect which is under investigation.

In the case of Maxwellian distributions, the average collision frequencies can be written as follows:

$$
\begin{aligned}
v_{i} & =2 \sqrt{2 \pi} a^{2} n_{d 0} v_{T i}\left(1+\chi_{i}\right), \\
v_{e} & =2 \sqrt{2 \pi} a^{2} n_{d 0} v_{T e} e^{\chi_{e}},
\end{aligned}
$$

where $\chi_{i} \equiv Z_{d} e^{2} /\left(a T_{i}\right), \quad \chi_{e} \equiv-\left(T_{i} / T_{e}\right) \chi_{i}, \quad$ and $v_{T \beta}=$ $\left(T_{\beta} / m_{\beta}\right)^{1 / 2}$.

Using these average collision frequencies and performing the calculation of the $\widehat{I}_{\beta}^{0}$ integral, the dispersion relation given by equation (4) can be written in the form

$$
1+2 \sum_{\beta} X_{\beta} \zeta_{\beta} \hat{\zeta}_{\beta}\left[1+\hat{\zeta}_{\beta} Z\left(\hat{\zeta}_{\beta}\right)\right]=0
$$


where $Z$ is the plasma dispersion function [24], defined by

$$
\begin{gathered}
Z(\zeta)=\frac{1}{\sqrt{\pi}} \int_{-\infty}^{+\infty} d t \frac{e^{-t^{2}}}{t-\zeta}, \\
\zeta_{\beta} \equiv \frac{\omega}{\sqrt{2} k_{\|} v_{T \beta}}, \quad \hat{\zeta}_{\beta}^{s} \equiv \frac{\omega+i v_{\beta}}{\sqrt{2} k_{\|} v_{T \beta}} .
\end{gathered}
$$

At this point it is useful to prepare the dispersion relation for the numerical solution, introducing the following dimensionless quantities:

$$
\begin{gathered}
z=\frac{\omega}{\omega_{p e 0}}, \quad q=\frac{k_{\|} c_{s}}{\omega_{p e 0}}, \quad \varepsilon=\frac{n_{d 0}}{n_{i 0}}, \\
u_{\beta}=\frac{v_{T \beta}}{c_{s}}, \quad \gamma=\frac{\lambda^{2} n_{i 0} c_{s}}{\omega_{p e 0}}, \quad \tilde{a}=\frac{a}{\lambda}, \lambda=\frac{e^{2}}{T_{i}}, \\
\tau_{e}=\frac{T_{e}}{T_{i}}, \quad, \quad \tilde{v}_{\beta}=\frac{v_{\beta}}{\omega_{p e 0}}, \quad r_{\beta}=\frac{\omega_{p \beta}}{\omega_{p e 0}},
\end{gathered}
$$

where $c_{S}$ is the ion-acoustic velocity,

$$
c_{s}=\sqrt{\frac{T_{e}}{m_{i}}},
$$

and where $\omega_{p e 0}$ is the electron plasma angular frequency evaluated considering the limiting situation $n_{d 0}=0$.

The relevant results can therefore be cast in terms of these dimensionless quantities. For instance, the dimensionless collision frequencies are given by:

$$
\begin{gathered}
\tilde{\mathrm{v}}_{i}=2 \sqrt{2 \pi} \varepsilon \gamma \tilde{a}^{2} u_{i}\left(1+\chi_{i}\right), \\
\tilde{\mathrm{v}}_{e}=2 \sqrt{2 \pi} \varepsilon \gamma \tilde{a}^{2} u_{e} e^{\chi_{e}},
\end{gathered}
$$

where now $\chi_{i}=Z_{d} / \tilde{a}$ and $\chi_{e}=-\chi_{i} / \tau_{e}$.

The dispersion relation, in terms of these dimensionless quantities, becomes

$$
1+\sum_{\beta} \frac{r_{\beta}^{2}}{q^{2} u_{\beta}^{2}}\left(1+i \frac{\tilde{v}_{\beta}}{z}\right)\left[1+\hat{\zeta}_{\beta} Z\left(\hat{\zeta}_{\beta}\right)\right]=0,
$$

where

$$
\hat{\zeta}_{\beta}=\frac{z+i \tilde{v}_{\beta}}{\sqrt{2} q u_{\beta}}
$$

Considering the case of $\left|\hat{\zeta}_{\beta}\right|>>1$, for $\beta=e, i$, we obtain the dispersion relation for Langmuir waves, modified by the presence of the dust,

$$
1+\sum_{\beta} \frac{r_{\beta}^{2}}{q^{2} u_{\beta}^{2}}\left(1+i \frac{\tilde{v}_{\beta}}{z}\right)\left[i \sqrt{\pi} \hat{\zeta}_{\beta} e^{-\hat{\zeta}_{\beta}^{2}}-\frac{1}{2 \hat{\zeta}_{\beta}^{2}}-\frac{3}{4 \hat{\zeta}_{\beta}^{4}}\right]=0 .
$$

If the effect of incorporation of charged plasma particles by the dust particles is neglected in the dispersion relation by the assumption of $\tilde{v}_{\beta}=0$, approximation frequently employed in the literature, it is easy to show that for $\left|z_{i}\right|<<\left|z_{r}\right|$, where $z_{r}$ and $z_{i}$ are the real and imaginary parts of $z$, and for $q^{2} u_{e}^{2}<<1$, the roots of the dispersion are given as follows,

$$
\begin{gathered}
z_{i} \simeq-\sqrt{\frac{\pi}{8}} \frac{r_{e}^{4}}{q^{3} u_{e}^{3}} e^{-3 / 2} \exp \left(-r_{e}^{2} / 2 q^{2} u_{e}^{2}\right), \\
z_{r} \simeq \pm r_{e}\left(1+\frac{3}{2} \frac{q^{2} u_{e}^{2}}{r_{e}^{2}}\right) .
\end{gathered}
$$

In the absence of dust, $r_{e}=1$, these expressions reduce to the familiar expressions for Langmuir waves, easily found in textbooks [25].

Another interesting case to be considered is that of $\left|\hat{\zeta}_{e}\right|<<$ 1 and $\left|\hat{\zeta}_{i}\right|>>1$, leading to the dispersion relation for ionacoustic waves, expected to occur for $\tau_{e}>>1$,

$$
\begin{gathered}
1+\frac{r_{e}^{2}}{q^{2} u_{e}^{2}}\left(1+i \frac{\tilde{\mathrm{v}}_{e}}{z}\right)\left[i \sqrt{\pi} \hat{\zeta}_{e} e^{-\hat{\zeta}_{e}^{2}}+1\right. \\
\left.-2 \hat{\zeta}_{e}^{2}\left(1-\frac{2}{3} \hat{\zeta}_{e}^{2}+\frac{4}{15} \hat{\zeta}_{e}^{4}\right)\right] \\
+\frac{r_{i}^{2}}{q^{2} u_{i}^{2}}\left(1+i \frac{\tilde{\mathrm{v}}_{i}}{z}\right)\left[i \sqrt{\pi} \hat{\zeta}_{i} e^{-\hat{\zeta}_{i}^{2}}-\frac{1}{2 \hat{\zeta}_{i}^{2}}-\frac{3}{4 \hat{\zeta}_{i}^{4}}\right]=0 .
\end{gathered}
$$

If the effect of absorption of plasma particles by the dust particles is neglected in Eq. (12), and if it is assumed that $\left|z_{i}\right|<<\left|z_{r}\right|$, the roots of the dispersion are given as follows,

$$
\begin{gathered}
z_{i} \simeq-\sqrt{\frac{\pi}{8} \frac{\left|z_{r}\right|^{4}}{q^{3}}}\left[\tau_{e}^{3 / 2} \exp \left(-\frac{\tau_{e} \alpha}{2\left(\alpha r_{e}^{2}+q^{2}\right)}\right)+\alpha^{1 / 2} r_{e}^{2}\right] \\
z_{r} \simeq \pm\left(\frac{\alpha q^{2}}{\alpha r_{e}^{2}+q^{2}}\right)^{1 / 2}
\end{gathered}
$$

where $\alpha=m_{e} / m_{i}$. In the absence of dust, $r_{e}=1$, these expressions reduce to the familiar expressions for ion-acoustic waves [25].

The expression for $z_{r}$ in Eq. (13) is equivalent to Eq. (40) of Ref. [11]. The imaginary part $z_{i}$ in Eq. (13) corresponds to the first term of Eq. (41) of Ref. [11], if the term corresponding to the ion damping in Eq. (13) is neglected. Eq. (41) in Ref. [11] displays a second term, which incorporates effects due to charging of dust particles due to collisions with plasma particles, and which is originated from a contribution to the dielectric tensor which contains a derivative of the charging currents, Eq. (19) of Ref. [11]. This kind of term corresponds in our case to the contribution due to $\varepsilon_{33}^{N}$, neglected in the present investigation. However, the dispersion relation utilized for the numerical analysis of ion-acoustic waves in the present investigation, Eq. (12), takes into account other effects due to the collisional charging of dust particles, appearing in the $\varepsilon_{33}^{C}$ contribution, which have been neglected in Ref. [11]. 


\section{NUMERICAL ANALYSIS OF LANGMUIR WAVES}

We consider the following parameters, which are in the range of parameters of interest for stellar winds: ion temperature $T_{i}=1.0 \times 10^{4} \mathrm{~K}$, ion density $n_{i 0}=1.0 \times 10^{9} \mathrm{~cm}^{-3}$, ion charge number $Z_{i}=1.0$, and ion mass $m_{i}=m_{p}$, the proton mass. For the radius of the dust particles, we assume $a=1.0 \times$ $10^{-4} \mathrm{~cm}$. For the classical distance of minimum approach, measured in $\mathrm{cm}$, we use the value $\lambda=1.44 \times 10^{-7} / T_{i}(\mathrm{eV})$, where $T_{i}(\mathrm{eV})$ means the ion temperature expressed in units of eV.

Initially, we examine the case of the high-frequency oscillations with $\omega \simeq \omega_{p e}$, known as Langmuir waves, and numerically search for the roots of Eq. (10), considering $T_{e}=T_{i}$. In Figs. 1(a) and 1(b) we show respectively the real and imaginary parts of the normalized frequency $\left(z_{r}\right.$ and $\left.z_{i}\right)$, as a function of the normalized wave number $q$, for $\varepsilon=0$. For comparison we also show the curves corresponding to the approximated analytical solution given by Eqs. (11), which are very close to the roots obtained numerically, for small values of $q$. It is noticed that Landau damping is negligible for small $q$, becoming meaningful only after $q \simeq 5.0 \times 10^{-3}$.

The effect of the dust on the Langmuir waves is illustrated in Figs. 2 and 3. Fig. 2 shows the value of $z_{r}$ as a function of $q$ and $\varepsilon$, considering $\varepsilon$ changing from 0.0 up to $1.0 \times 10^{-4}$. It is seen that the quantity $z_{r}$ appears to be quite insensitive to the presence of the dust. In Fig. 3 we see the corresponding imaginary part. In the scale of the figure, the quantity $z_{i}$ also appears to be insensitive to the presence of the dust. However, an amplified view of the large wavelength region (small $q$ ), where Landau damping is negligible, shows the occurrence of damping due to the presence of the dust particles. Fig. 4(a) shows the absolute value of $z_{i}$ for values of $q$ between $3.2 \times$ $10^{-3}$ and $4.2 \times 10^{-3}$. It is seen that for $\varepsilon=0.0$ values of $\left|z_{i}\right|$ due to Landau damping of order $10^{-6}$ starts to appear for $q \simeq 0.004$. In the region of smaller $q$ (larger wavelengths), the damping rate is zero for $\varepsilon=0.0$, but it is seen to increase with the increase of the dust density. Fig. 4(a) shows that the damping due to the dust particles increases linearly with the dust density, reaching the maximum of $\left|z_{i}\right| \simeq 6.0 \times 10^{-6}$ for the maximum value of $\varepsilon$ considered in the calculation.

In Fig. 4(b) we show the value of $\left|z_{i}\right|$ obtained from Eq. (10), if the damping effect due to the collisional charging of the dust particles is neglected, that is, if we assume $\tilde{\mathrm{v}}_{e}=\tilde{\mathrm{v}}_{i}=$ 0 . The range depicted in Fig. 4(b) is the same range shown in Fig. 4(a). The damping at large wavelengths $(q<0.4)$ shown by Fig. 4(a) does not appear in Fig. 4(b), indicating that it is entirely due to the effect of the collisional charging of the dust particles.

Another view of this effect of the absorption of charged plasma particles by dust particles is seen in Figs. 5(a) and $5(\mathrm{~b})$, which show the values of $z_{i}$ as a function of the normalized wave number $q$, for five values of $\varepsilon$. Fig. 5(a) shows the values of $z_{i}$ obtained from numerical solution of the full Eq. (10), including the effect of the collisional charging which appears in $\tilde{\mathrm{v}}_{e}$ and $\tilde{\mathrm{v}}_{i}$. Considering the curve obtained for $\varepsilon=0.0$, it is seen that Landau damping becomes meaningful only at $q \simeq 0.004$. For increasing values of $\varepsilon$, Fig. 5(a) displays
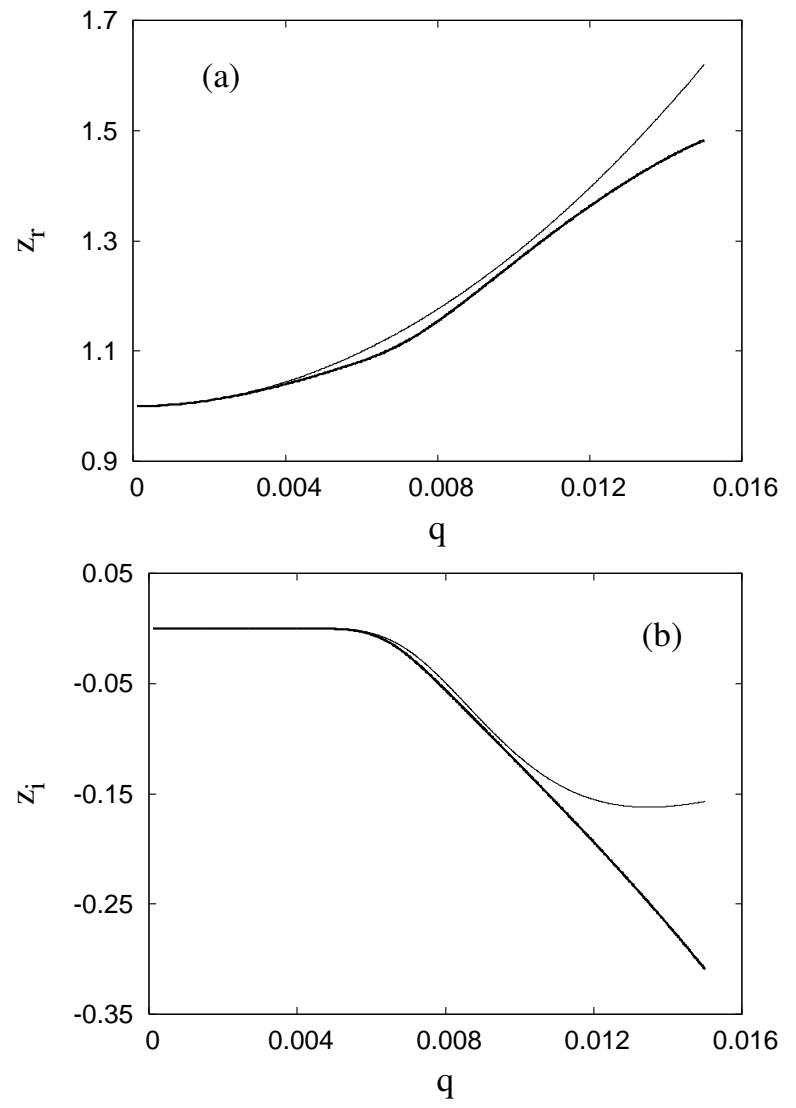

FIG. 1: Real and imaginary parts of the normalized frequency corresponding to Langmuir waves, obtained from Eq. (10) for $\varepsilon=0$. The thin lines gives the results obtained from the approximated analytical solution given by Eq. (11). (a) $z_{r}$ as a function of $q$; (b) $z_{i}$ as a function of $q . T_{i}=1.0 \times 10^{4} \mathrm{~K}, n_{i 0}=n_{e 0}=1.0 \times 10^{9} \mathrm{~cm}^{-3}, Z_{i}=1.0$, $m_{i}=m_{p}$, and $T_{e}=T_{i}$.

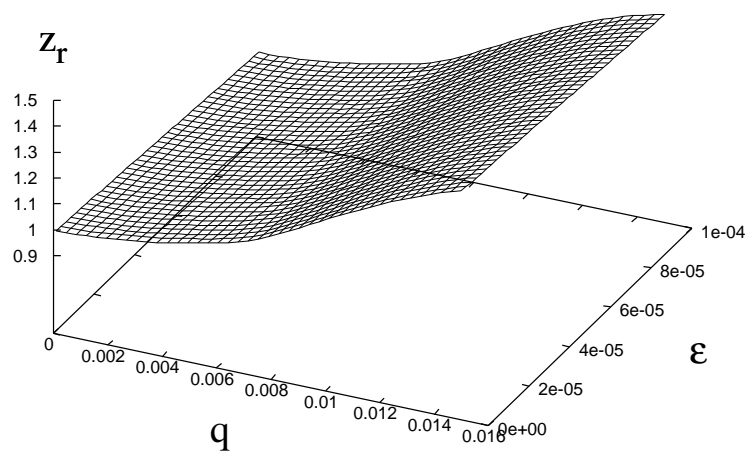

FIG. 2: Real part of the normalized frequency $\left(z_{r}\right)$ for the Langmuir waves, as a function of $q$ and $\varepsilon$. Radius of dust particles, $a=1.0 \times$ $10^{-4} \mathrm{~cm}$. Other parameters as in Fig. 1. 


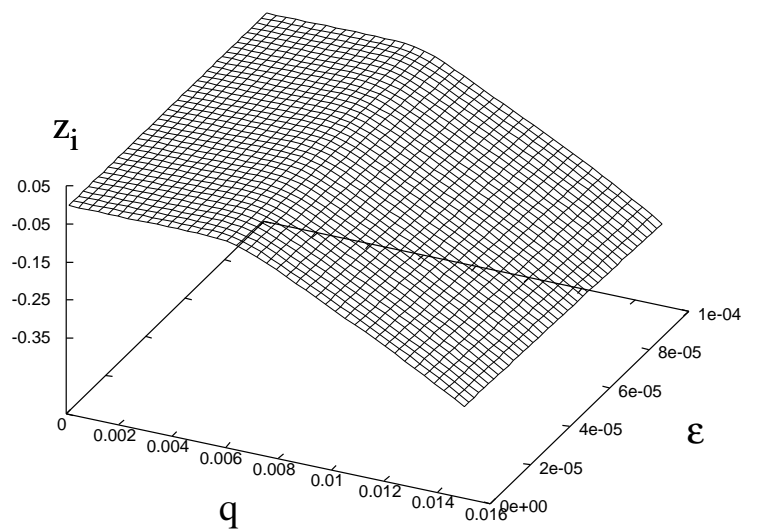

FIG. 3: Imaginary part of the normalized frequency $\left(z_{i}\right)$ for the Langmuir waves, as a function of $q$ and $\varepsilon$. Radius of dust particles, $a=1.0 \times 10^{-4} \mathrm{~cm}$. Other parameters as in Fig. 1 .
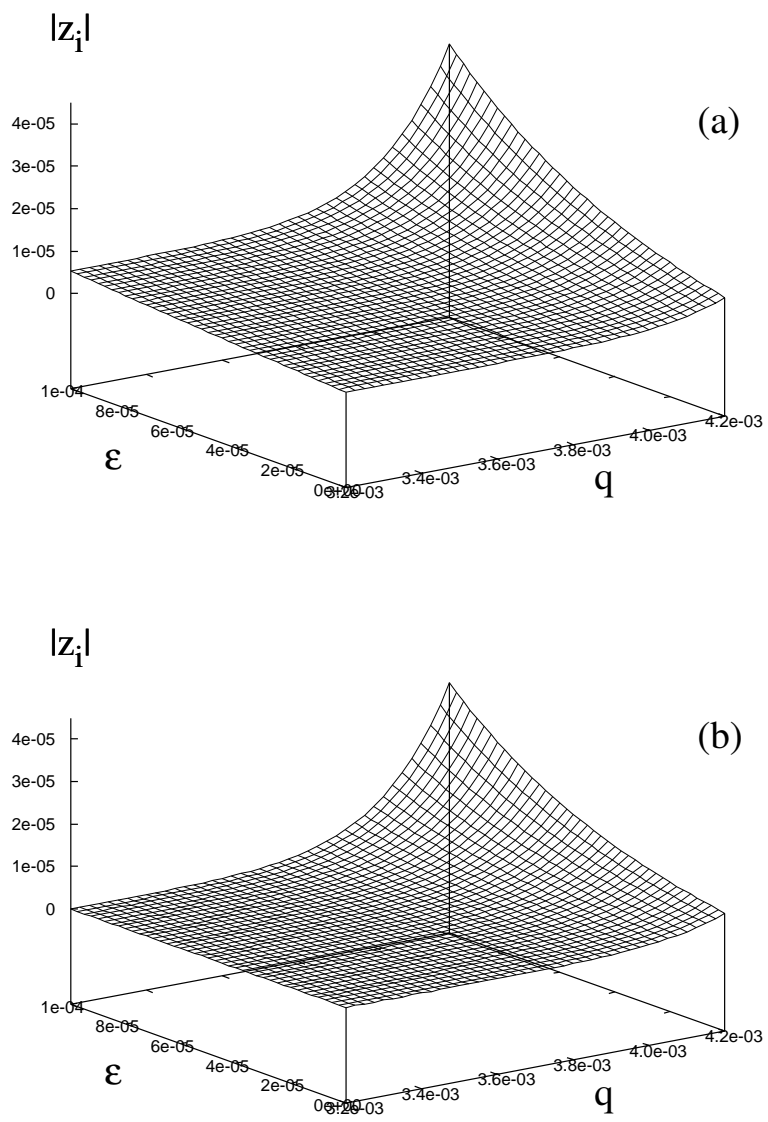

FIG. 4: (a) Amplified view of $\left|z_{i}\right|$ for the Langmuir waves, as a function of $q$ and $\varepsilon$; (b) amplified view of $\left|z_{i}\right|$ for the Langmuir waves, obtained with $\tilde{\mathrm{v}}_{i}=\tilde{\mathrm{v}}_{e}=0$ in Eq. (10). The parameters are the same as in Fig. 2.
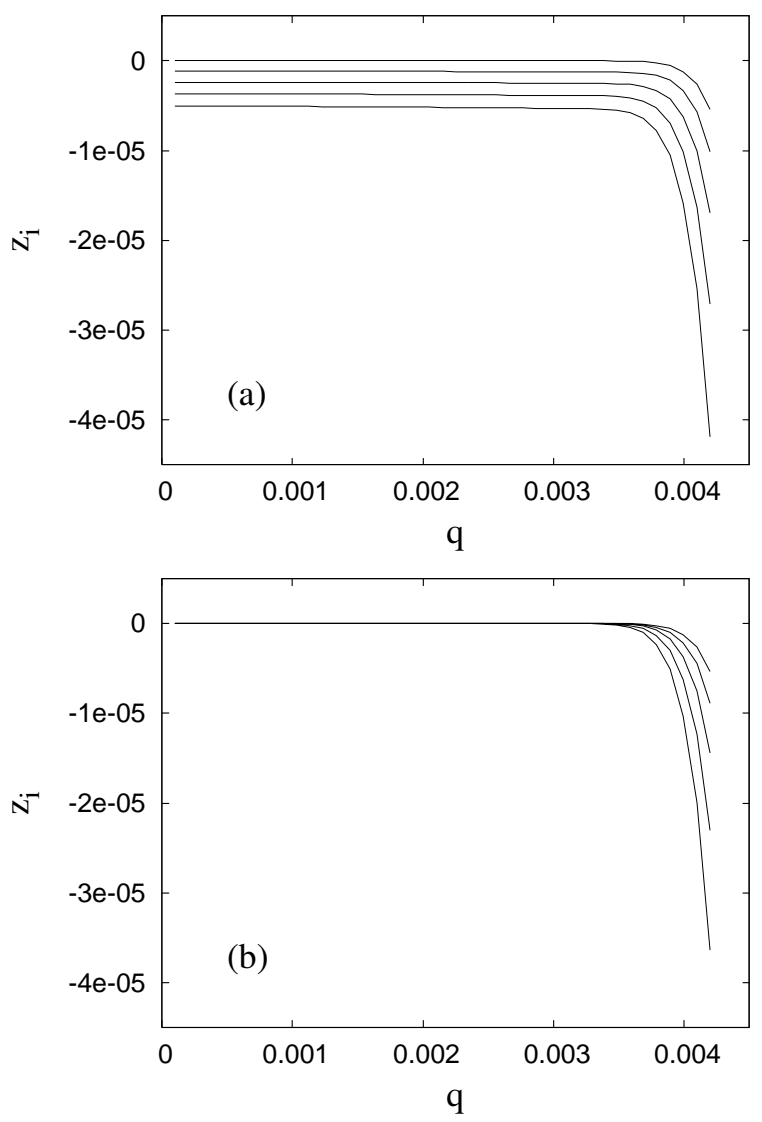

FIG. 5: $z_{i}$ for the Langmuir waves as a function of $q$, for five values of $\varepsilon\left(\varepsilon=0.0,2.5 \times 10^{-5}, 5.0 \times 10^{-5}, 7.5 \times 10^{-5}\right.$, and $\left.1.0 \times 10^{-4}\right)$. (a) From the numerical solution of Eq. (10); (b) From the numerical solution obtained with $\tilde{\mathrm{v}}_{i}=\tilde{\mathrm{v}}_{e}=0$ in Eq. (10). The parameters are the same as in Fig. 2.

clearly the appearance of a damping rate practically independent of $q$, for $q<0.004$. On the other hand, if the effect of collisional charging is neglected in Eq. (10), by taking $\tilde{v}_{e}=\tilde{v}_{i}=0.0$, the curves depicted in Fig. 5(b) are obtained. It is seen that the Landau damping at $q \geq 0.004$ is modified by the presence of the dust, reflecting the change of the electron population. However, the damping for large-wavelength waves (small values of $q$ ) is completely absent in Fig. 5(b), corroborating the previous conclusion that it is entirely due to the effect of incorporation of charged plasma particles by the dust particles, via inelastic collisions.

\section{NUMERICAL ANALYSIS OF ION-ACOUSTIC WAVES}

We now examine the case of the low-frequency oscillations, with $\omega<<\omega_{p e}$, known as ion-acoustic waves, and numerically search for the roots of Eq. (12), considering $T_{e}=20 T_{i}$, and other parameters as in Section IV. This value of electron temperature is considered as a representative example, since ion-acoustic waves are expected to occur for $T_{e}>>T_{i}$, 

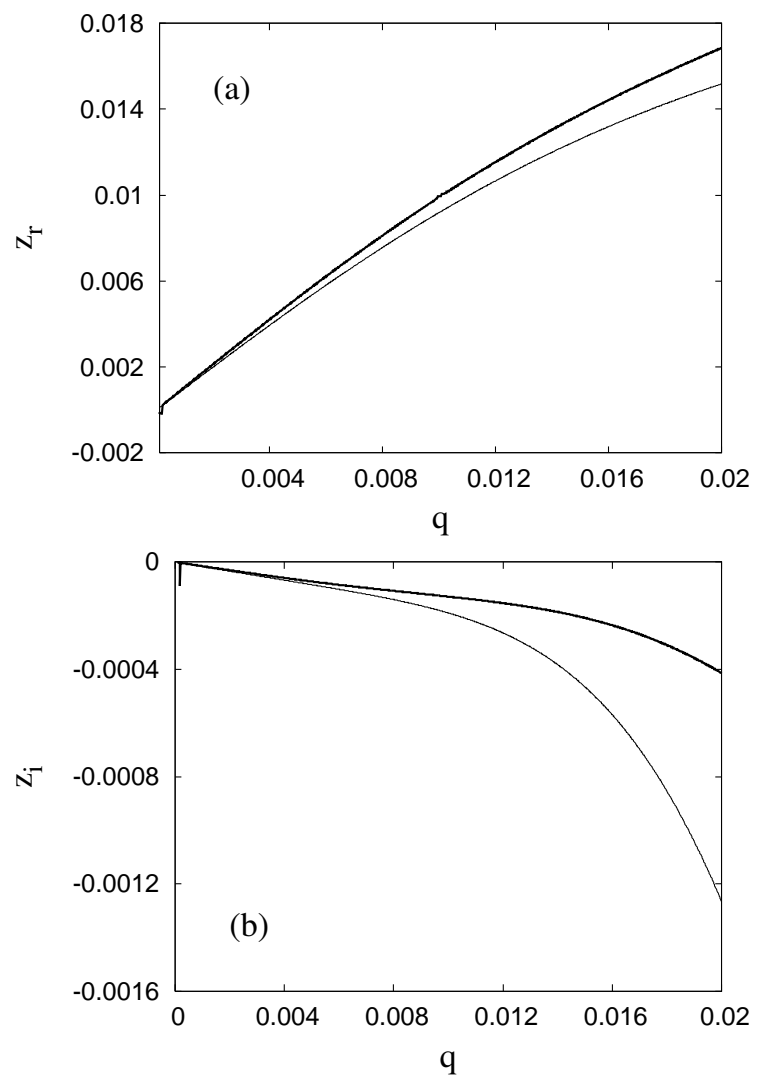

FIG. 6: Real and imaginary parts of the normalized frequency corresponding to ion-acoustic waves, obtained from Eq. (12) for $\varepsilon=0$. The thin lines gives the results obtained from the approximated analytical solution given by Eq. (13). (a) $z_{r}$ as a function of $q$; (b) $z_{i}$ as a function of $q$. The parameters are the same as in Fig. 2.

at least in dustless plasmas. In Figs. 6(a) and 6(b) we show respectively the real and imaginary parts of the normalized frequency $\left(z_{r}\right.$ and $\left.z_{i}\right)$, as a function of the normalized wave number $q$, for $\varepsilon=0$. For comparison we also show the curves corresponding to the approximated analytical solution given by Eqs. (13). It is seen that the outcome of the analytical solution is very close to the root obtained numerically, for small values of $q$. Landau damping of weak intensity $\left(\left|z_{i}\right|<1.0 \times 10^{-3}\left|z_{r}\right|\right)$ occurs even for very small $q$, namely for large wavelength waves.

The effect of the dust on the ion-acoustic waves is shown in Figs. 7 and 8. Fig. 7 shows the value of $z_{r}$ for ion-acoustic waves as a function of $q$ and $\varepsilon$, considering $\varepsilon$ changing from 0.0 up to $1.0 \times 10^{-4}$. It is seen that the quantity $z_{r}$ appears to be quite insensitive to the presence of the dust, similarly to the behavior found for $z_{r}$ in the case of Langmuir waves. In Fig. 8 we see the corresponding imaginary part. The dependence of this quantity on the dust density appears to be quite complex. Fig. 8 shows that the magnitude of the damping rate is increased as effect of the presence of the dust, for large wavelength waves $(q \leq 0.015$, with the most significant effect occurring for $q \simeq 0.005$ ). On the other hand, for $q$ closer to

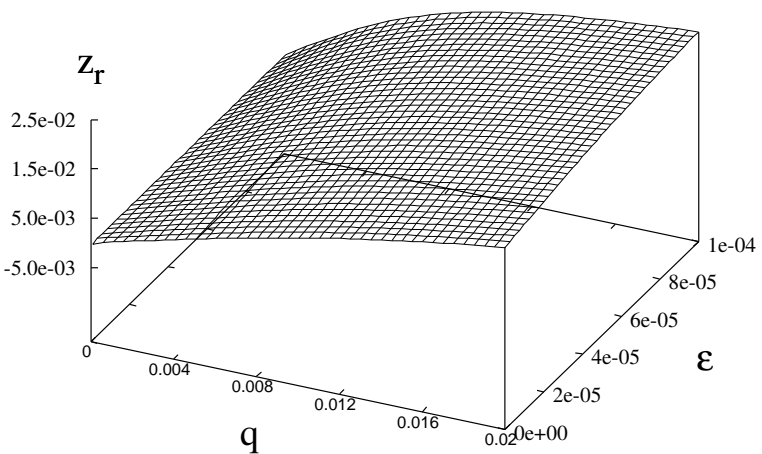

FIG. 7: Real part of the normalized frequency $\left(z_{r}\right)$ for the ionacoustic waves, as a function of $q$ and $\varepsilon$. The parameters are the same as in Fig. 2.

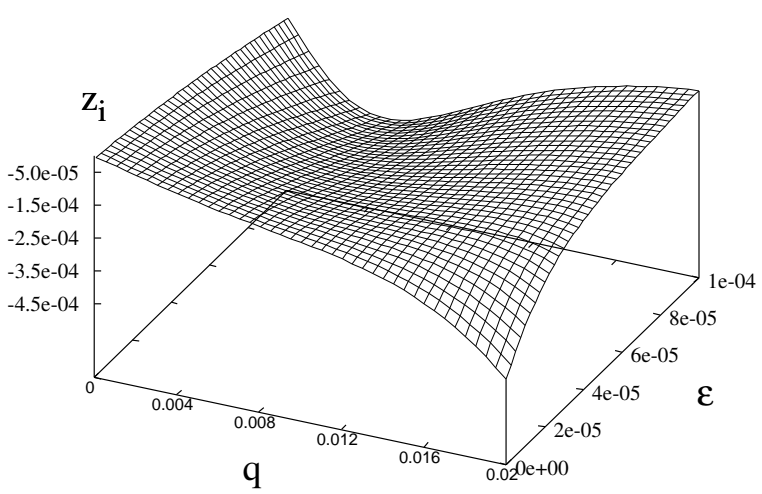

FIG. 8: Imaginary part of the normalized frequency $\left(z_{i}\right)$ for the ionacoustic waves, as a function of $q$ and $\varepsilon$. The parameters are the same as in Fig. 2.

0.02, where significant Landau damping occurs for $\varepsilon=0.0$, the presence of the dust contributes to decrease the magnitude of the damping.

In Fig. 9 we compare the values of $z_{i}$ obtained from the dispersion relation given by Eq. (12) with those obtained from the dispersion relation obtained when the effects of collisional charging of dust particles are neglected by the assumption of $\tilde{\mathrm{v}}_{e}=\tilde{\mathrm{v}}_{i}=0$. Fig. 9 shows that an important effect of the presence of the dust on the damping of ion-acoustic waves is due to the modification of the charge-neutrality condition, contrarily to what we have obtained in the case of Langmuir waves. It is seen that for ion-acoustic waves, if the effect of collisional charging is neglected, the damping rate is quantitatively modified, but the overall qualitative effect of the presence of the dust remains basically unchanged.

The different behavior of the dependence of the damping rate with the dust density, for Langmuir and ion-acoustic waves, may be explained as follows. The Landau damping occurs due to the effect of the imaginary part of the $Z$ function, which contains the terms proportional to $\exp \left(-\hat{\zeta}_{\beta}^{2}\right)$. These 

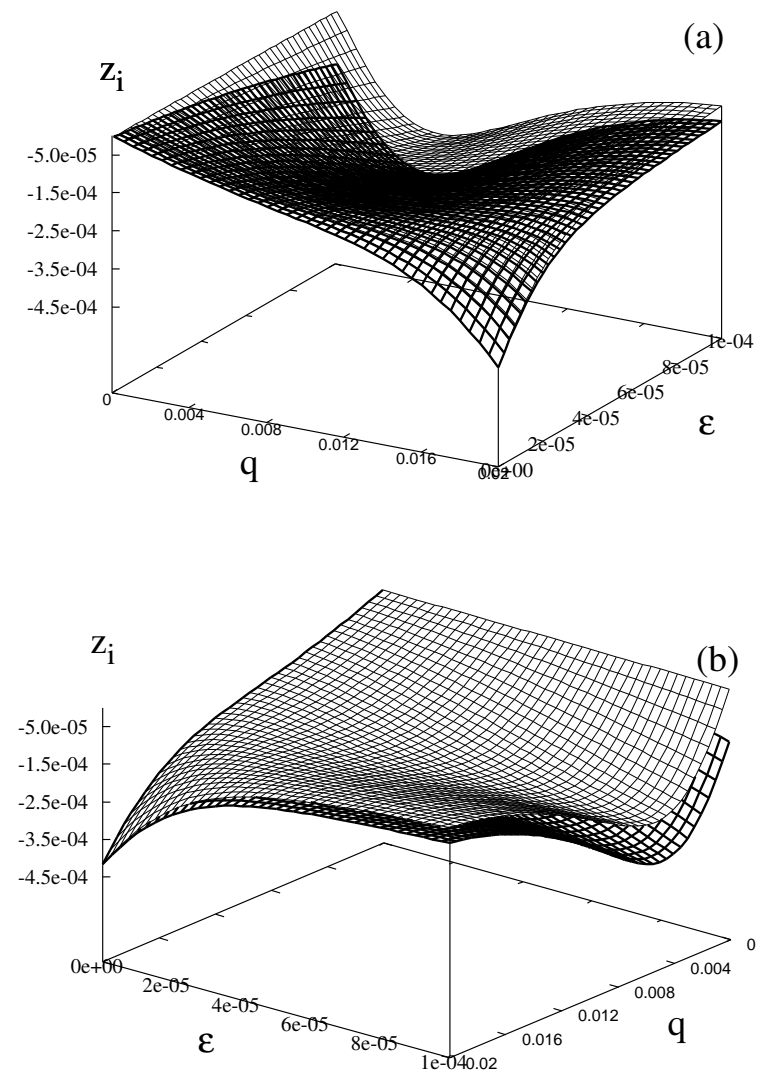

FIG. 9: Imaginary part of the normalized frequency $\left(z_{i}\right)$ for the ionacoustic waves, as a function of $q$ and $\varepsilon$. The surface with thick lines is obtained from the numerical solution of Eq. (12), and the surface with thin lines is obtained from the same dispersion relation, with $\tilde{\mathrm{v}}_{i}=\tilde{\mathrm{v}}_{e}=0$. Panels (a) and (b) display the quantity from two different points of view. The parameters are the same as in Fig. 2.

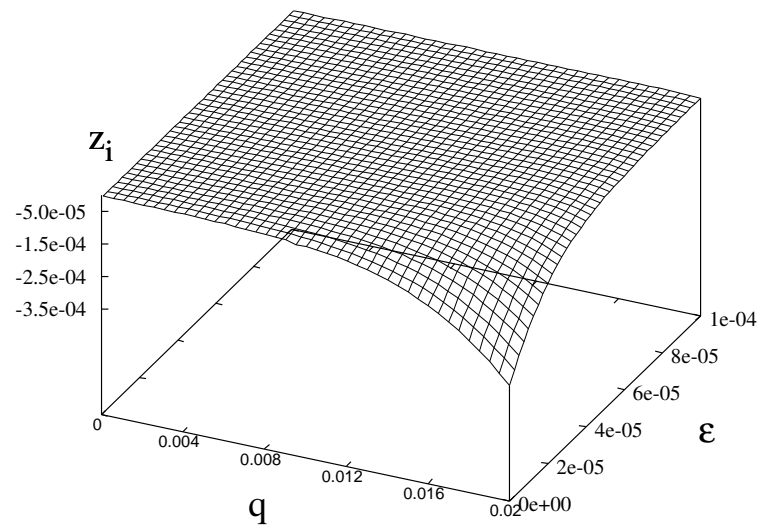

FIG. 10: Imaginary part of the normalized frequency $\left(z_{i}\right)$ for the ionacoustic waves, as a function of $q$ and $\varepsilon$, obtained from Eq. (12) with $\tilde{v}_{i}=\tilde{v}_{e}=0$, by neglecting the term with $\left(i \sqrt{\pi} \hat{\zeta}_{e} \exp \left(-\hat{\zeta}_{e}^{2}\right)\right)$. The parameters are the same as in Fig. 2.
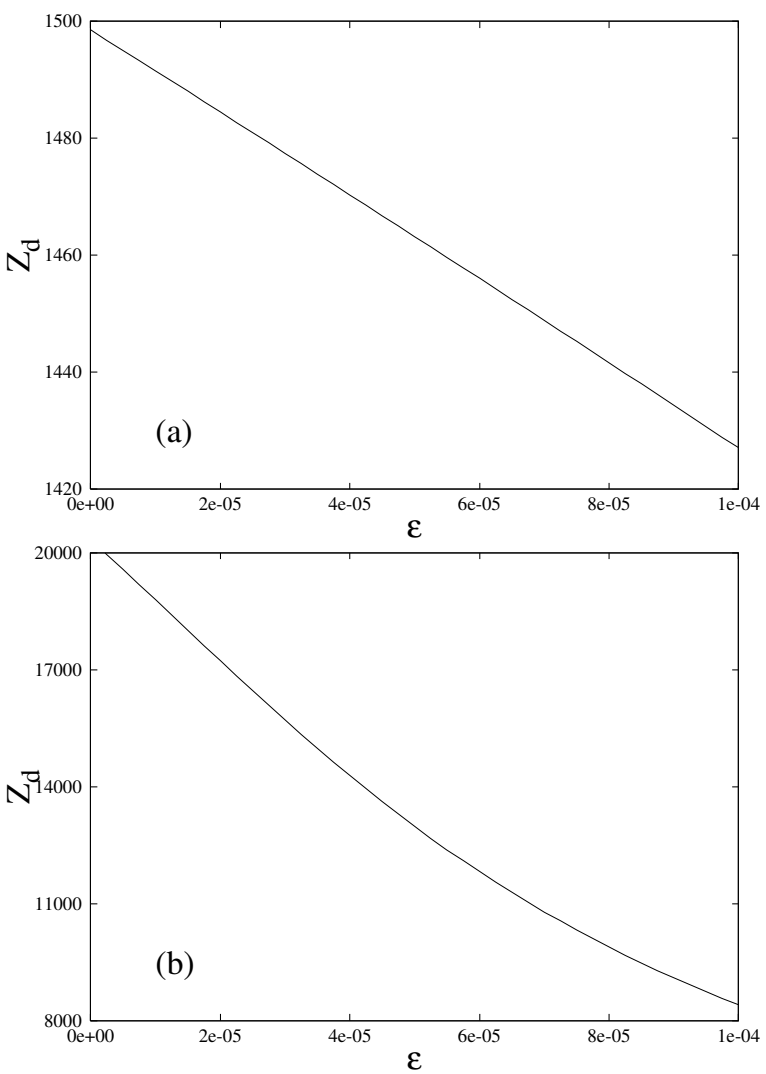

FIG. 11: (a) $Z_{d}$ as a function of $\varepsilon$, for $T_{e}=T_{i}$. (b) $Z_{d}$ as a function of $\varepsilon$, for $T_{e}=20 T_{i}$. Other parameters as in Fig. 2.

terms depend on the density of particles of the respective species, ions and electrons. In the case of Langmuir waves, we have $\left|\hat{\zeta}_{e}\right|>>1$ and $\left|\hat{\zeta}_{i}\right|>>1$, causing negligible Landau damping for small $q$. The Landau damping only starts to become meaningful for the larger values of $q$ considered in Fig. 4, and is entirely due to the electron population, which is changed with the presence of the dust. These considerations explain the behavior depicted in Figs. 4(b) and 5(b), namely the occurrence of damping only for the upper range of the values of $q$ depicted in the figures, with the damping rate depending on the dust density. On the other hand, if the collisional absorption of electrons and ions by dust particles is taken into account via the contributions of $\tilde{\mathrm{v}}_{e}$ and $\tilde{\mathrm{v}}_{i}$ in Eq. (10), there is an additional source for the imaginary part of the dispersion relation, which is non-vanishing even for small $q$. The outcome are the curves exhibited in Figs. 4(a) and 5(a), which show non-vanishing values of $z_{i}$ for very small $q$, in the presence of dust.

On the other hand, the dispersion relation for ion-acoustic waves is obtained under the condition $\left|\hat{\zeta}_{i}\right|>>1$ and $\left|\hat{\zeta}_{e}\right|<<1$. The term originated from the imaginary part of the $Z$ function is present also for the small values of $q$, originating Landau damping even if the contribution of the collisional charging of dust particles is neglected. The value of damping is of course dependent on the electron density, and therefore on the dust 

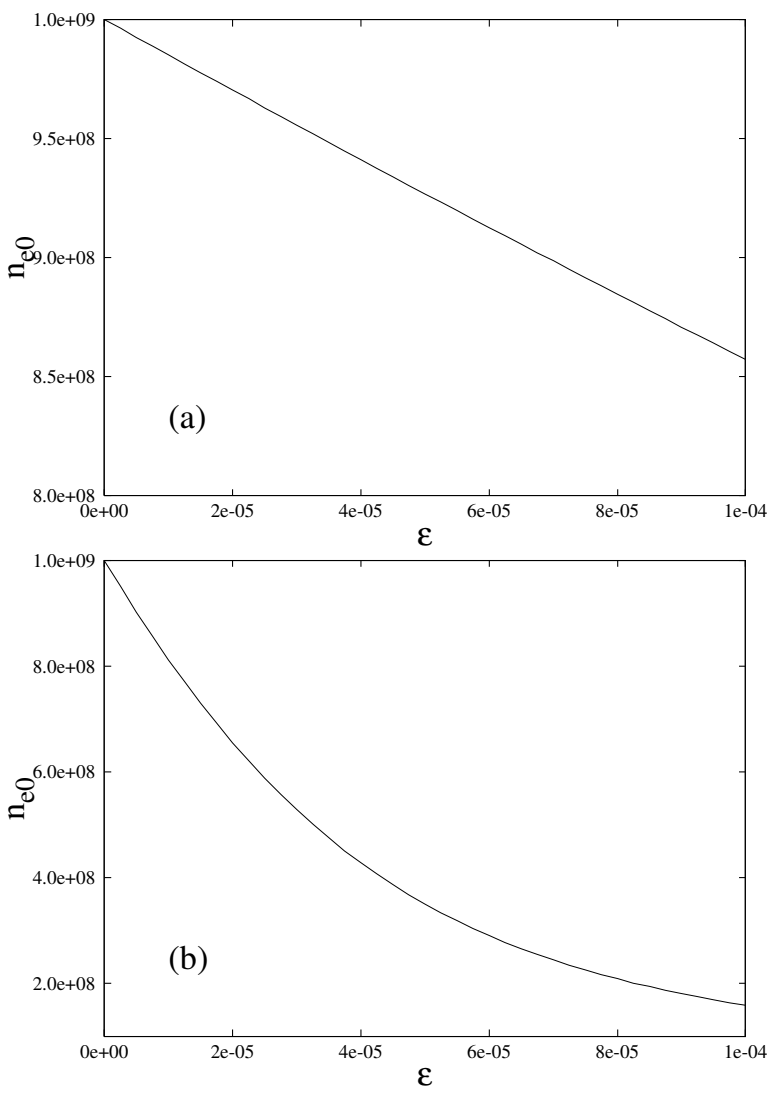

FIG. 12: (a) $n_{e 0}$ as a function of $\varepsilon$, for $T_{e}=T_{i}$. (b) $n_{e 0}$ as a function of $\varepsilon$, for $T_{e}=20 T_{i}$. Other parameters as in Fig. 2.

density. When the effect of collisional charging is taken into account, the magnitude of the damping is modified, but the overall qualitative features remain. These features can be observed in Fig. 9. Further verification of this explanation can be found in Fig. 10, which shows $z_{i}$ obtained from Eq. (12) when the effect of collisional charging is neglected, and when the term proportional to $\exp \left(-\hat{\zeta}_{e}^{2}\right)$ is made artificially to vanish. That is, in addition to neglecting the effect of the absorption of charged particles by the dust, the effect of electron Landau damping is neglected. As expected from the previous reasoning, the outcome is a root in which the imaginary part $z_{i}$ vanishes for small $q$. For the larger values of $q$ occurs Landau damping due to the ions, which is also reduced in magnitude with the increase of the dust density. These features can be understood by the analysis of the analytical expression for the damping of ions-acoustic waves, Eq. (13). If the electron Landau damping is neglected, the approximate expression for the damping of the ion-acoustic waves is reduced to

$$
z_{i} \simeq-\sqrt{\frac{\pi}{8}} \frac{\left|z_{r}\right|^{4}}{q^{3}}\left[\tau_{e}^{3 / 2} \exp \left(-\frac{\tau_{e} \alpha}{2\left(\alpha r_{e}^{2}+q^{2}\right)}\right)\right] .
$$

It is easily seen that for increasing dust density, which means decreasing values of $r_{e}$, the value of $\left|z_{i}\right|$ is reduced, as displayed by the numerical solution shown in Fig. 10.
An important additional information may be obtained by considering the dependence of the electron density and of the equilibrium dust charge, on the dust density. In Figs. 11 and 12 we show respectively the values of the dust charge $Z_{d}$ and of the electron density $n_{e 0}$ as a function of $\varepsilon$, for two values of the ratio $T_{e} / T_{i}$. Fig. 11(a) shows the value of $Z_{d}$ for $T_{e}=T_{i}$, the ratio of temperature significant for Langmuir waves, and Fig. 11(b) shows the value of $Z_{d}$ for $T_{e}=20 T_{i}$, representative for the case of ion-acoustic waves. It is seen that in the case of $T_{e}=T_{i}$ the value of $Z_{d}$ changes by less than $5 \%$ when $\varepsilon$ is changed between 0.0 and $1.0 \times 10^{-4}$, and changes by $60 \%$ in the case $T_{e}=20 T_{i}$. In Figs. 12(a) and 12(b) it is also seen that the value of $n_{e 0}$ is decreased by approximately $15 \%$ when $\varepsilon$ is changed between 0.0 and $1.0 \times 10^{-4}$, in the case of $T_{e}=T_{i}$, and is reduced to approximately $15 \%$ of the original value in the case $T_{e}=20 T_{i}$. These results show that the electron density and the dust charge are more and more sensitive to the dust density for increasing values of the ratio $T_{e} / T_{i}$, for fixed ion density.

\section{CONCLUSIONS}

In the present paper we have used a kinetic description to analyze wave propagation in dusty plasmas, taking into account the incorporation of electrons and ions to the dust particles, due to inelastic collisions. We have considered the case of wave propagation exactly parallel to the external magnetic field, and Maxwellian distributions for electrons and ions in the equilibrium situation, and we have derived a dispersion relation for electrostatic waves in the dusty plasma, which is exactly the same as the dispersion relation for electrostatic waves in unmagnetized dusty plasmas. This dispersion relation has been utilized for investigation of Langmuir waves, with $\omega \simeq \omega_{p e}$, considering $T_{e}=T_{i}$, and also for investigation of the low-frequency waves denominated ion-acoustic waves, considering $T_{e}=20 T_{i}$.

The results obtained have shown the appearance of a small damping effect for large wavelength Langmuir waves, due to the collisional charging of the dust particles. We have seen that this damping effect at large wavelengths vanishes if the collisional absorption of charged plasma particles by dust particles is neglected. The reason is that, if the collisional charging is neglected, the influence of the dust particles occurs only via the charge imbalance between electrons and ions. The electron density is therefore modified by the presence of the dust, affecting the Landau damping, which is anyway negligible for large wavelengths.

We have also analyzed the dependence of the damping rate of ion-acoustic waves on the wavelength and on the dust density. Our results have shown the complex dependence of this damping rate on the dust density, indicating that the damping due to the electronic population may increase for relatively high wavelengths, due to the presence of the dust, comparatively with the case without dust. The results have also shown that the damping of ion-acoustic waves is dependent on the dust population even if the effect of collisional incorporation of electrons and ions by dust grains is not taken into account, 
a behavior differing from that of the damping of Langmuir waves.

It is noticed that the present numerical investigation did not show the appearance of instability due to the presence of the dust, either for Langmuir waves or for ion-acoustic waves. The dust particles contribute to wave absorption because they suffer inelastic collisions from ions and electrons, in the dissipative process of dust charging. This absence of instability in the numerical analysis corroborates analytical results obtained with a fluid formulation [2], contrasting with some earlier and somewhat controversial results indicating instability of longitudinal oscillations due to the collisional charging of dust particles $[7,8,26]$.

Our results have been obtained with the use of an apparently sound approximation, which uses the average value of the inelastic collision frequencies of electrons and ions with the dust particles, instead of the actual momentum dependent expressions. We intend to investigate the effect of this approximation, reporting our findings in a forthcoming publication.

Another further development to be introduced in the formulation would be the incorporation to the numerical analysis of a novel term to be added to the dielectric tensor, entirely due to the effect of dust charge fluctuations. In the formulation utilized in the present paper, and in other studies available in the literature, the conventional dielectric tensor is utilized, modified by the presence of the dust. The need for the additional term, already demonstrated by theoretical analysis, [11, 1719, 27], has hitherto been ignored in the numerical analysis. Our results regarding the effect of this additional term are expected to appear in the near future.

\section{APPENDIX A: THE COMPONENTS OF THE DIELECTRIC TENSOR FOR A HOMOGENEOUS MAGNETIZED DUSTY PLASMA}

We assume that the distribution function of particles of species $\beta$, in a dusty plasma, satisfies Vlasov's equation appended with a term describing binary collisions with dust particles,

$$
\begin{array}{r}
\frac{\partial f_{\beta}}{\partial t}+\frac{\mathbf{p}}{m_{\beta}} \cdot \nabla f_{\beta}+q_{\beta}\left[\mathbf{E}+\frac{\mathbf{p}}{m_{\beta} c} \times \mathbf{B}\right] \cdot \nabla_{\mathbf{p}} f_{\beta} \\
=-\int \sigma_{\beta} \frac{p}{m_{\beta}}\left(f_{d} f_{\beta}-f_{d 0} f_{\beta 0}\right) d q,
\end{array}
$$

where $f_{d 0}$ and $f_{\beta 0}$ represent respectively the equilibrium distribution functions of dust particles and of particles of species $\beta$, with the subscript $\beta=e, i$ identifying electrons and ions, respectively. The distribution function for the dust particles, $f_{d}$, satisfies the following equation,

$$
\frac{\partial f_{d}}{\partial t}+\frac{\partial}{\partial q}\left[I(\mathbf{r}, q, t) f_{d}\right]=0
$$

where

$$
I(\mathbf{r}, q, t)=\sum_{\beta} \int d^{3} p q_{\beta} \sigma_{\beta}(p, q) \frac{p}{m_{\beta}} f_{\beta}(\mathbf{r}, \mathbf{p}, t),
$$

is the current of electrons and ions which charge the dust particles [10]. The presence of the collisional term in these equations assures the possibility of variation of the electric charge of the dust particles, due to the inelastic collisions with particles of species $\beta$.

Upon linearization, the perturbed distribution function satisfies the following equation,

$$
\begin{aligned}
& \frac{\partial f_{\beta 1}}{\partial t}+\frac{\mathbf{p}}{m_{\beta}} \cdot \nabla f_{\beta 1}+q_{\beta}\left(\frac{\mathbf{p}}{m_{\beta} c} \times \mathbf{B}_{0}\right) \cdot \nabla_{\mathbf{p}} f_{\beta 1}+v_{\beta d}^{0}(p) f_{\beta 1} \\
& =-v_{\beta d}^{1}(\mathbf{r}, p, t) f_{\beta 0}-q_{\beta}\left[\mathbf{E}_{1}+\frac{\mathbf{p}}{m_{\beta} c} \times \mathbf{B}_{1}\right] \cdot \nabla_{\mathbf{p}} f_{\beta 0},
\end{aligned}
$$

where

$$
\begin{aligned}
v_{\beta d}^{0}(p) & =\int_{-\infty}^{0} \sigma_{\beta}(p, q) \frac{p}{m_{\beta}} f_{d 0}(q) d q, \\
v_{\beta d}^{1}(\mathbf{r}, p, t) & =\int_{-\infty}^{0} \sigma_{\beta}(p, q) \frac{p}{m_{\beta}} f_{d 1}(\mathbf{r}, q, t) d q,
\end{aligned}
$$

and $\sigma_{\beta}$ is the charging cross-section, given by [28]

$$
\sigma_{\beta}=\pi a^{2}\left(1-\frac{2 q_{d} q_{\beta} m_{\beta}}{a p^{2}}\right) H\left(1-\frac{2 q_{d} q_{\beta} m_{\beta}}{a p^{2}}\right) .
$$

After use of Fourier-Laplace transform in the system of equations describing the dusty plasmas, the perturbed distribution function can be written as [18]:

$$
\hat{f}_{\beta}(\mathbf{p})=\hat{f}_{\beta}^{C}+\hat{f}_{\beta}^{N}
$$

where

$$
\begin{gathered}
\hat{f}_{\beta}^{C}=-q_{\beta} \int_{-\infty}^{0} d \tau e^{i\left\{\mathbf{k} \cdot \mathbf{R}-\left[\omega+i v_{\beta d}^{0}(p)\right] \tau\right\}} \\
\times\left(\hat{\mathbf{E}}+\frac{1}{m_{\beta} \gamma_{\beta} c} \mathbf{p}^{\prime} \times \hat{\mathbf{B}}\right) \cdot \nabla_{\mathbf{p}^{\prime}} f_{\beta 0}\left(p_{\perp}, p_{\|}\right), \\
\hat{f}_{\beta}^{N}=-\int_{-\infty}^{0} d \tau e^{i\left\{\mathbf{k} \cdot \mathbf{R}-\left[\omega+i v_{\beta d}^{0}(p)\right] \tau\right\}} \hat{v}_{\beta d}(p) f_{\beta 0} .
\end{gathered}
$$

One notices that $\hat{f}_{\beta}^{C}$ has the same formal structure as the perturbed distribution obtained in the evaluation of the dielectric tensor of a conventional homogeneous magnetized plasma, with $\omega+i v_{\beta d}^{0}(p)$ instead of $\omega$ in the argument of the exponential function. This part of the perturbed distribution therefore gives rise to a contribution to the components of the dielectric tensor that corresponds to the usual form of the components obtained for dustless magnetized homogeneous plasma, except for the modifications due to the presence of the inelastic collision frequency $v_{\beta d}^{0}(p)$, which is related to the equilibrium distribution function of dust particles, as shown by Eq. (A3). On the other hand, $\hat{f}_{\beta}{ }^{N}$ contains the inelastic collision frequency $\hat{v}_{\beta d}$ in the numerator of the integral over 
the equilibrium distribution function, and vanishes in the case of dustless plasma. The $\hat{v}_{\beta d}$ quantity is the Fourier-Laplace transform of the $v_{\beta d}^{1}$ collision frequency, related to collisions with the fluctuating distribution of dust particles.

Using these two contributions to the perturbed distribution function, the dielectric tensor for a magnetized dusty plasma, homogeneous, fully ionized, with identical immobile dust particles and charge variable in time, could be written in the following way $[18,19]$

$$
\varepsilon_{i j}=\varepsilon_{i j}^{C}+\varepsilon_{i j}^{N}
$$

One notices that the separation in the two terms appearing in Eq. (A6) should not be considered arbitrary, since it is motivated by the different nature of the two contributions to the perturbed distribution function depicted by Eq. (A5).

The term $\varepsilon_{i j}^{C}$ is formally identical, except for the $i 3$ components, to the dielectric tensor of a magnetized homogeneous conventional plasma of electrons and ions, with the resonant denominator modified by the addition of a purely imaginary term which contains the inelastic collision frequency of dust particles with electrons and ions. For the $i 3$ components of the dielectric tensor, in addition to the term obtained with the prescription above, there is a term which is proportional to this inelastic collision frequency. The expression for $\varepsilon_{i j}^{C}$ is $[18,19]$

$$
\begin{gathered}
\varepsilon_{i j}^{C}=\delta_{i j}+\sum_{\beta} \frac{X_{\beta}}{n_{\beta 0}} \\
\times \sum_{n=-\infty}^{+\infty} \int d^{3} p p_{\perp} \frac{\varphi_{0}\left(f_{\beta 0}\right)}{D_{n \beta}}\left(\frac{p_{\|}}{p_{\perp}}\right)^{\delta_{i 3}+\delta_{j 3}} R_{i j}^{n \beta} \\
-\delta_{i 3} \delta_{j 3} \sum_{\beta} \frac{X_{\beta}}{n_{\beta 0}} \int d^{3} p L\left(f_{\beta 0}\right) \frac{p_{\|}}{p_{\perp}}
\end{gathered}
$$

$$
+\delta_{j 3} \sum_{\beta} \frac{X_{\beta}}{n_{\beta 0}} \sum_{n=-\infty}^{+\infty} \int d^{3} p\left[i \frac{v_{\beta d}^{0}(p)}{\omega} \frac{L\left(f_{\beta 0}\right)}{D_{n \beta}}\left(\frac{p_{\|}}{p_{\perp}}\right)^{\delta_{i 3}}\right] R_{i j}^{n \beta},
$$

where

$$
\begin{gathered}
D_{n \beta}=1-\frac{k_{\|} p_{\|}}{m_{\beta} \omega}-\frac{n \Omega_{\beta}}{\omega}+i \frac{v_{\beta d}^{0}(p)}{\omega}, \\
R_{11}^{n \beta}=\frac{n^{2}}{b_{\beta}^{2}} J_{n}^{2}\left(b_{\beta}\right), \quad R_{33}^{n \beta}=J_{n}^{2}\left(b_{\beta}\right), \\
R_{12}^{n \beta}=-R_{21}^{n \beta}=i \frac{n}{b_{\beta}} J_{n}\left(b_{\beta}\right) J_{n}^{\prime}\left(b_{\beta}\right), \\
R_{13}^{n \beta}=R_{31}^{n \beta}=\frac{n}{b_{\beta}} J_{n}^{2}\left(b_{\beta}\right), \quad R_{22}^{n \beta}=J_{n}^{\prime 2}\left(b_{\beta}\right),
\end{gathered}
$$

$$
\begin{gathered}
R_{23}^{n \beta}=-R_{32}^{n \beta}=-i J_{n}\left(b_{\beta}\right) J_{n}^{\prime}\left(b_{\beta}\right), \\
v_{\beta d}^{0}(p)=\frac{\pi a^{2} n_{d 0}}{m_{\beta}} \frac{\left(p^{2}+C_{\beta}\right)}{p} H\left(p^{2}+C_{\beta}\right), \\
\varphi_{0}\left(f_{\beta 0}\right)=\frac{\partial f_{\beta 0}}{\partial p_{\perp}}-\frac{k_{\|}}{m_{\beta} \omega} L\left(f_{\beta 0}\right), \\
L\left(f_{\beta 0}\right)=p_{\|} \frac{\partial f_{\beta 0}}{\partial p_{\perp}}-p_{\perp} \frac{\partial f_{\beta 0}}{\partial p_{\|}}, \\
X_{\beta}=\frac{\omega_{p \beta}^{2}}{\omega^{2}}, \quad \omega_{p \beta}^{2}=\frac{4 \pi n_{\beta 0} q_{\beta}^{2}}{m_{\beta}}, \quad \Omega_{\beta}=\frac{q_{\beta} B_{0}}{m_{\beta} c}, \\
b_{\beta}=\frac{k_{\perp} p_{\perp}}{m_{\beta} \Omega_{\beta}}, \quad C_{\beta}=-\frac{2 q_{\beta} m_{\beta} q_{d 0}}{a},
\end{gathered}
$$

where $q_{d 0}=\varepsilon_{d} e Z_{d}$ is the equilibrium charge of the dust particle (positive, $\varepsilon_{d}=+1$, or negative, $\varepsilon_{d}=-1$ ) and $H$ denotes the Heaviside function.

The term $\varepsilon_{i j}^{N}$ is entirely new and arises only due to the process of fluctuation of the charge of the dust particles. Its form is strongly dependent on the model used to describe the charging process of the dust particles. The expression for this term is $[18,19]$

$$
\varepsilon_{i j}^{N}=-\frac{4 \pi i n_{d 0}}{\omega} U_{i} S_{j}
$$

with

$$
\begin{aligned}
U_{i} \equiv & \frac{i}{\omega+i\left(v_{c h}+v_{1}\right)} \sum_{\beta} \frac{q_{\beta}}{m_{\beta}^{2}} \\
& \times \sum_{n=-\infty}^{+\infty} \int d^{3} p \frac{p_{\perp} p \sigma_{\beta}^{\prime}(p) f_{\beta 0}}{D_{n \beta}}\left(\frac{p_{\|}}{p_{\perp}}\right)^{\delta_{i 3}} R_{i 3}^{n \beta}, \\
S_{j} & \equiv \frac{1}{\omega n_{d 0}} \sum_{\beta} q_{\beta}^{2} \sum_{n=-\infty}^{+\infty} \int d^{3} p \frac{v_{\beta d}^{0}(p)}{\omega} \\
& \times \frac{\varphi_{0}\left(f_{\beta 0}\right)}{D_{n \beta}}\left(\frac{p_{\|}}{p_{\perp}}\right)^{\delta_{j 3}} R_{3 j}^{n \beta} \\
& -\frac{\delta_{j 3}}{\omega n_{d 0}} \sum_{\beta} q_{\beta}^{2} \int d^{3} p \frac{v_{\beta d}^{0}(p)}{\omega} \frac{L\left(f_{\beta 0}\right)}{p_{\perp}} \\
& +i \frac{\delta_{j 3}}{\omega n_{d 0}} \sum_{\beta} q_{\beta}^{2} \sum_{n=-\infty}^{+\infty} \int d^{3} p \frac{\left[v_{\beta d}^{0}(p) / \omega\right]^{2}}{D_{n \beta}} \\
& \times \frac{L\left(f_{\beta 0}\right)}{p_{\perp}} R_{3 j}^{n \beta},
\end{aligned}
$$

where

$$
v_{c h}=-\sum_{\beta} \frac{q_{\beta}}{m_{\beta}} \int d^{3} p p \sigma_{\beta}^{\prime}(p) f_{\beta 0},
$$




$$
\begin{aligned}
v_{1} & =\sum_{\beta} \frac{q_{\beta}}{m_{\beta}} \sum_{n=-\infty}^{+\infty} \int d^{3} p \frac{\left[i v_{\beta d}^{0}(p) / \omega\right]}{D_{n \beta}} \\
& \times p \sigma_{\beta}^{\prime}(p) f_{\beta 0} R_{33}^{n \beta} .
\end{aligned}
$$

In the expressions (A9), (A11) and (A12), we have used the notation $\left.\sigma_{\beta}^{\prime}(p) \equiv\left(\partial \sigma_{\beta} / \partial q_{d}\right)\right|_{q_{d}=-Z_{d} e}$, and $\sigma_{\beta}$ is given by Eq. (A4).

Effects of the collisional charging of the dust particles occur in the terms with $v_{\beta d}^{0}(p) / \omega$, and effects of presence of dust particles, introduced via quasi-neutrality relation $\left(n_{i 0} \neq n_{e 0}\right)$, occurs in terms with $X_{\beta} \equiv \omega_{p \beta}^{2} / \omega^{2}$.

\section{APPENDIX B: COMPARING THE FORMULATION OF THE PRESENT PAPER WITH SOME RESULTS FOUND IN THE LITERATURE}

In this appendix it is established the connection between the expressions for the dielectric tensor which we are using, based on the formulation developed in Ref. [18] and given in its general form in Appendix A, and some expressions very used in the literature. In particular we show that our results can be cast in the form which appears in a series of papers of Tsytovich et al. [10, 29, 30], if restrictive assumptions are made.

We start with the general expressions of Appendix A, and concentrate in the 33 component of the dielectric tensor, which is the only component which is of interest for the present comparison, and we make the same restrictions used by Tsytovich at al. in the cited works. According to Eq. (A6), we write this component as

$$
\varepsilon_{33}=\varepsilon_{33}^{C}+\varepsilon_{33}^{N} .
$$

Assuming the absence of external magnetic field and concentrating in the case of electrostatic oscillations, it is seen that only the term $n=0$ contributes to the sum in $n$ in equation (A7) of Appendix A, and that terms which contain $L\left(f_{\beta 0}\right)$ are absent. Then we can write

$$
\varepsilon_{33}^{C}=1+\chi_{33}
$$

where

$$
\chi_{33}=\frac{1}{\omega} \sum_{\beta} \frac{4 \pi q_{\beta 0}^{2}}{k_{\|}} \int d^{3} p \frac{k_{\|} v_{\|}}{\omega-k_{\|} v_{\|}+i v_{\beta d}^{0}(v)} \frac{\partial f_{\beta 0}}{\partial p_{\|}} .
$$

Upon completion of the numerator of the integrand and some further manipulations, we obtain

$$
\begin{gathered}
\chi_{33}=\sum_{\beta} \frac{4 \pi q_{\beta 0}^{2}}{k_{\|}^{2}} \int d^{3} p \frac{1}{\omega-k_{\|} v_{\|}+i v_{\beta d}^{0}(v)} \mathbf{k} \cdot \frac{\partial f_{\beta 0}}{\partial \mathbf{p}} \\
+\frac{1}{\omega} \sum_{\beta} \frac{4 \pi q_{\beta 0}^{2}}{k_{\|}^{2}} \int d^{3} p \frac{i v_{\beta d}^{0}(v)}{\omega-k_{\|} v_{\|}+i v_{\beta d}^{0}(v)} \mathbf{k} \cdot \frac{\partial f_{\beta 0}}{\partial \mathbf{p}} .
\end{gathered}
$$

In order to compare with Ref. [30] we must replace $k_{\|} \rightarrow k$ and $f_{\beta 0} \rightarrow \Phi_{\mathbf{p}}^{\beta}$. Noting that $v_{\beta d}^{0}(v)$ is equal to $v_{d, \beta}(v)$, given by equation (5) of Ref. [30], and considering that in our model neutral particles are not included in the formulation, $\mathrm{v}_{n, \beta}=0$, it is seen that $v_{\beta}(v)$ used in Ref. [30], is the same as $v_{\beta d}^{0}(v)$ of the present paper. After some trivial manipulations, it is therefore possible to write as follows the $\varepsilon_{33}^{C}$ component of the present formulation,

$$
\varepsilon_{33}^{C}=1+\sum_{\beta} \chi_{\mathbf{k}, \omega}^{\beta}+\frac{n_{d}}{\omega} \chi_{\mathbf{k}, \omega}^{\sigma},
$$

where $\chi_{\mathbf{k}, \omega}^{\beta}$ and $\chi_{\mathbf{k}, \omega}^{\sigma}$ are given by Eqs. (20) and (21) of Ref. [30].

Using the same assumptions it is easy to obtain similar results relevant to the term $\varepsilon_{33}^{N}$. For instance, for $j=3$ Eq. (A10) can be cast as follows,

$$
S_{3}=-i \frac{k_{\|}}{4 \pi \omega} \chi_{\mathbf{k}, \omega}^{\sigma}
$$

Moreover, from (A11) it is seen that $\mathrm{v}_{c h}$ is the same used in Ref. [30], and from Eq. (A12), we obtain

$$
v_{1}=i n_{d} \chi_{\mathbf{k}, \omega}^{\sigma, \sigma^{\prime}}
$$

where $\chi_{\mathbf{k}, \omega}^{\sigma, \sigma^{\prime}}$ is given by Eq. (23) of Ref. [30].

Therefore, it is seen that for $i=3 \mathrm{Eq}$. (A9) can be written in the following form,

$$
U_{3}=\frac{i \omega}{\omega+i\left(v_{c h}+v_{1}\right)} \sum_{\beta} \int d^{3} p \frac{v v_{\|} \sigma_{\beta}^{\prime} f_{\beta 0}}{\omega-k_{\|} v_{\|}+i v_{\beta d}^{0}} .
$$

Inserting all these results in Eq. (A8) and re-arranging terms, we get

$$
\varepsilon_{33}^{N}=\chi_{\mathbf{k}, \omega}^{d e i}-\frac{n_{d}}{\omega} \chi_{\mathbf{k}, \omega}^{\sigma},
$$

where $\chi_{\mathbf{k}, \omega}^{d e i}$ is given by equation (30) of Ref. [30].

Therefore it is seen that the complete 33 component of the dielectric tensor, derived using the formulation of the present paper, is given by

$$
\varepsilon_{33}=1+\sum_{\beta} \chi_{\mathbf{k}, \omega}^{\beta}+\chi_{\mathbf{k}, \omega}^{d e i} .
$$

Taking into account that in the formulation of the present paper the dynamics of the dust particles is not included, it is seen that Eq. (B1) is the same as Eq. (28) of Ref. [30].

\section{ACKNOWLEDGMENTS}

M. C. de Juli was supported by Fundação de Amparo à Pesquisa do Estado de São Paulo (FAPESP) (process number: 01/10921-9). L. F. Ziebell and V. Jatenco-Pereira acknowledge support from Conselho Nacional de Desenvolvimento Científico e Tecnológico $(\mathrm{CNPq})$. The work was also supported by Fundação de Amparo à Pesquisa no Estado do Rio Grande do Sul (FAPERGS), Programa/Convênio PRONEX/CNPq. 
[1] P. K. Bliokh and V. V. Yarashenko, Soviet. Astr. Engl. Transl. 29, 330 (1985).

[2] J. R. Bhatt, Physical Review E 55, 1166 (1997).

[3] N. D’Angelo, Planet. Space Sci. 42, 507 (1994).

[4] R. K. Varma, P. K. Shukla, and V. Krishan, Physical Review E 47, 3612 (1993).

[5] M. R. Jana, A. Sen, and P. K. Kaw, Physical Review E 48, 3930 (1993).

[6] J. X. Ma and M. Y. Yu, Physics of Plasmas 1, 3520 (1994).

[7] F. Li, O. Havnes, and F. Melands $\varnothing$, Planet. Space Sci. 42, 401 (1994).

[8] J. R. Bhatt and B. P. Pandey, Physical Review E 50, 3980 (1994).

[9] S. V. Vladimirov, K. N. Ostrikov, M. Y. Yu, and L. Stenflo, Physical Review E 58, 8046 (1998).

[10] V. N. Tsytovich and O. Havnes, Comm. Plasma Phys. Contr. Fus. 15, 267 (1993).

[11] S. I. Popel, S. N. Andreev, A. A. Gisko, A. P. Golub, and T. V. Losseva, Plasma Physics Reports 8, 284 (2004).

[12] M. Rosenberg, Planet. Space Sci. 41, 229 (1993).

[13] X. Liang, J. Zheng, J. X. Ma, W. D. Liu, J. Xie, G. Zhuang, and C. X. Yu, Physics of Plasmas 8, 1459 (2001).

[14] A. Barkan, N. D' Angelo, and R. L. Merlino, in International Conference on Physics of Dusty Plasma, 1996. Goa (World Scientific, Singapore, 1997), Vol. Advances in Physics of Dusty Plasmas, Chap. Laboratory studies of ion and dust acoustic instabilities in dusty plasmas, pp. 30-40, ed. P. H. Shukla, D. A. Mendis and T. Desay.

[15] A. Barkan, N. D’Angelo, and R. L. Merlino, Planet. Space Sci.
44, 239 (1996).

[16] A. Barkan, N. D’Angelo, and R. L. Merlino, Physics Letters A 222, 329 (1996)

[17] M. C. de Juli, R. S. Schneider, L. F. Ziebell, and V. JatencoPereira, Physics of Plasmas 12, 052109 (2005).

[18] M. C. de Juli and R. S. Schneider, J. Plasma Phys. 60, 243 (1998).

[19] M. C. de Juli and R. S. Schneider, J. Plasma Phys. 64, 57 (2000).

[20] J. S. Chang and K. Spariosu, J. Phys. Soc. Japan 62, 97 (1993).

[21] J. E. Allen, Physica Scripta 45, 497 (1992).

[22] V. N. Tsytovich, Sov. Phys. Usp. 40, 53 (1997).

[23] G. E. Morfill, H. M. Thomas, U. Konopka, and M. Zuzic, Physics of Plasmas 6, 1769 (1999).

[24] B. D. Fried and S. D. Conte, The Plasma Dispersion Function (Academic Press, New York, 1961).

[25] N. A. Krall and A. W. Trivelpiece, Principles of Plasma Physics (McGraw-Hill, New York, 1973).

[26] J.-X. Ma and M. Y. Yu, Physical Review E 50, R2431 (1994).

[27] M. C. de Juli, R. S. Schneider, D. A. Falceta-Gonçalves, and V. Jatenco-Pereira, IEEE Transactions on Plasma Science 32, 542 (2004).

[28] L. J. Spitzer, Physical Processes in the Interstellar Medium (John Wiley, New York, 1978).

[29] V. N. Tsytovich, U. de Angelis, and R. Bingham, Physical Review Letters 87, 185004, 4p. (2001).

[30] V. N. Tsytovich, U. de Angelis, and R. Bingham, Physics of Plasmas 9, 1079 (2002). 